\title{
Síndrome de ovario poliquístico
}

\section{Polycistic ovary syndrome}

\author{
Marlon A García-Santamaría 1,a , Juan A Viteri-Rodríguez 2,b
}

\section{RESUMEN}

El síndrome de ovario poliquístico (SOP) es la disfunción endocrino-metabólica más frecuente en la mujer, aparece generalmente después de la menarquia, se considera la principal causa de hiperandrogenismo (HA), los genes CYP19, CYP11a, CYP17, CYP21 y FST son susceptibles y han sido identificados como contribuyentes a esta enfermedad. Genera un desequilibrio hormonal de producción atípica de esteroides, estrógenos y andrógenos, impidiendo la ovulación con un crecimiento anormal de folículos, es causante de alteraciones en el ciclo menstrual, promueve la formación de quistes en los ovarios y el aumento de tamaño de estos, acné, hirsutismo, escaso desarrollo mamario, obesidad, infertilidad y cambios emocionales. Para el diagnóstico debe existir la presencia de 2 o 3 de los siguientes condicionantes: oligoanovulación crónica, polisquistosis ovárica por ecografía, hiperandrogenismo clínico o bioquímico; En cuanto al tratamiento, se recomienda utilizar anticonceptivos orales, citrato de clomifeno y metformina.

Palabras Clave: Hiperandrogenismo, poligénica, infertilidad, anticonceptivos orales, metformina.

\begin{abstract}
Polycystic ovary syndrome (POS) is the most common endocrine-metabolic dysfunction in women, it generally appears after menarche, it is considered the main cause of hyperandrogenism (HA), the CYP19, CYP11a, CYP17, CYP21 and FST genes are susceptible and have been identified as contributors to this disease. It generates a hormonal imbalance of atypical production of steroids, estrogens and androgens, preventing ovulation with an abnormal growth of follicles, causing alterations in the menstrual cycle, promoting the formation of cysts in the ovaries and their increase in size, acne, hirsutism, poor breast development, obesity, infertility and emotional changes. For the diagnosis, there must be the presence of 2 or 3 of the following conditions: chronic oligoanovulation, ovarian polyschistosis by ultrasound, clinical or biochemical hyperandrogenism; Regarding treatment, it is recommended to use oral contraceptives, clomiphene citrate and metformin.
\end{abstract}

Palabras Clave: hyperandrogenism, polygenic, infertility, contraceptives oral, metformin

\footnotetext{
${ }^{1}$ Facultad de Medicina. Universidad Regional Autónoma de los Andes “Uniandes”. Ecuador.

a Estudiante de Medicina.

${ }^{\mathrm{b}}$ Médico Genetista.
}

Marlon A García-Santamaría: ORCID: https://orcid.org/0000-0002-2331-733X

Juan A Viteri Rodríguez: ORCID: https://orcid.org/0000-0002-2463-7036

Correspondencia: Marlon A García-Santamaría

Correo: andres22med22@gmail.com 


\section{INTRODUCCIÓN}

El síndrome de ovario poliquístico (SOP) es la principal causa de hiperandrogenismo y una de las endocrinopatías más frecuentes en la mujer. Se presenta en la pubertad, cons-tituye una patología con una presentación clínica variable, en la que se encuentran diferentes fenotipos que resultan de la asociación de oligoanovulación, hiperandrogenismo y poliquistosis ovárica ecográfica. El diagnóstico síndrome de ovario poliquístico es de exclusión, en donde se descar-ta otras patologías que pueden presentar similar fenotipo clínico. La dificultad en su diagnóstico radica en la hetero-geneidad en su expresión clínica debido a los trastornos reproductivos y comorbilidades metabólicas que se asocian frecuentemente a este síndrome (diabetes tipo 2, obesidad, enfermedad cardiovascular, hiperplasia endometrial, inferti-lidad, apneas del sueño, entre otros). El tratamiento está di-rigido a reducir las manifestaciones del hiperandrogenismo, atender el trastorno ovulatorio, diagnosticar precozmente las comorbilidades, así como también mejorar el pronóstico reproductivo en las pacientes infértiles con SOP ${ }^{(1)}$.

\section{Epidemiología}

Establecer la prevalencia del síndrome de ovario poliquístico es difícil, depende de criterios diagnósticos. Los criterios más aceptados son los de Rotterdam, en donde se destaca el aspecto ecográfico de los ovarios. Hay que considerar que en un $15-20 \%$ de las mujeres sanas están presentes los ovarios de apariencia poliquística, en la época peripube-ral alcanzan una prevalencia del 30-40 Tabla 1. Epidemiologia de Síndrome de Ovario Poliquístico en pacientes de sexo femenino de entre 11 y 69 años atendidas

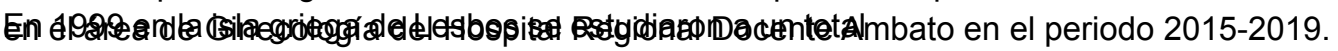

\begin{tabular}{|c|c|c|c|c|c|c|c|c|c|c|c|c|c|c|c|c|c|}
\hline \multirow[t]{2}{*}{$\begin{array}{l}\text { Edad } \\
\text { (años) }\end{array}$} & \multirow[t]{2}{*}{ Grupo } & \multicolumn{5}{|c|}{ Número } & \multicolumn{5}{|c|}{ Presuntivo } & \multicolumn{5}{|c|}{ Definitivo } & \multirow{2}{*}{$\begin{array}{l}\text { Casos definiti- } \\
\text { vos }(\%)\end{array}$} \\
\hline & & 2015 & 2016 & 2017 & 2018 & 2019 & 2015 & 2016 & 2017 & 2018 & 2019 & 2015 & 2016 & 2017 & 2018 & 2019 & \\
\hline 11 a 19 & $\begin{array}{l}\text { Adoles- } \\
\text { cencia }\end{array}$ & 2 & 4 & 11 & 7 & 10 & 0 & 1 & 4 & 3 & 7 & 2 & 3 & 7 & 4 & 3 & 10,67 \\
\hline 20 a 39 & Juventud & 11 & 10 & 36 & 32 & 30 & 1 & 0 & 10 & 12 & 9 & 10 & 10 & 26 & 20 & 19 & 47,75 \\
\hline 40 a 59 & Adultez & 12 & 7 & 26 & 22 & 20 & 0 & 1 & 7 & 9 & 5 & 12 & 6 & 19 & 13 & 15 & 36,51 \\
\hline 60 a 65 & Vejez & 0 & 0 & 2 & 2 & 6 & 0 & 0 & 0 & 0 & 1 & 0 & 0 & 2 & 2 & 5 & 5,05 \\
\hline TOTAL & & 25 & 21 & 75 & 63 & 66 & 1 & 2 & 21 & 24 & 22 & 24 & 19 & 54 & 39 & 42 & \\
\hline
\end{tabular}

Fuente: Base de datos del Hospital Regional Docente Ambato de pacientes atendidas en el área de ginecología en el periodo $2015-2019$. de 192 mujeres de entre 17 y 45 años, a las cuales dividieron en cuatro grupos. En el primero incluyeron a 108 mujeres sin alteración del ciclo menstrual ni hirsutismo, en el segundo a 56 pacientes con ciclo normal e hirsutismo, en el tercero a 10 mujeres con oligomenorrea sin hirsutismo y en el cuarto a 18 pacientes con oligomenorrea e hirsutismo. Calcularon una prevalencia del síndrome en la población estudiada de un $6,7 \%$ (2).

La influencia étnica en la prevalencia del SOP se evidenció en un estudio donde se observó el doble de prevalencia del síndrome en mujeres hispanas respecto a las afroamericanas en un área concreta de Estado Unidos, en Reino Unido utilizando criterios ecográficos se observó una prevalencia del síndrome en un $22 \%$ de mujeres caucásicas, frente a un $52 \%$ de mujeres asiáticas originarias de la India (2).

En la ciudad de Ambato, en el año 2019, en el Hospital Regional Docente Ambato se recolectaron datos de 250 pacientes desde el año 2015 al 2019 (tabla 1) con diagnóstico presuntivo y definitivo de síndrome de ovario poliquísticolos cuales se obtuvo como diagnóstico definitivo en 178 pacientes, encontrando una mayor incidencia en el grupo etario de mujeres jóvenes entre los 20 a 39 años seguida de mujeres adultas de 40 a 60 años. Por lo cual se determinó que el $73,2 \%$ de mujeres con SOP son diagnosticadas en su mayoría en la juventud y adultez ${ }^{(2)}$.

\footnotetext{
Fuente: Base de datos del Hospital Regional Docente Ambato de pacientes atendidas en el área de ginecología en el periodo $2015-2019$.
} 


\section{Fisiopatología}

EI SOP es una alteración metabólica-hormonal que se caracteriza por factores poligénico con influencias ambientales. Investigaciones demuestran que es una de las alteraciones más frecuentes entre las mujeres en edad fértil (3).

La glándula pituitaria llamada también hipófisis está localizada en el cerebro y produce la hormona luteinizante (LH), la hormona folículo estimulante (FSH), prolactina (PRL) y corticotropina. La fisiopatología del síndrome de ovario poliquístico, se destaca: un trastorno metabólico (resistencia insulínica e hiperinsulinemia), una disfunción neuroendocrina (hipersecreción de LH), y una disfunción de la esteroidogénesis y de la foliculogénesis ovárica (4).

Estas hormonas tienen un papel fundamental en el desequilibro hormonal y en causar el síndrome de ovario poliquístico.

\section{Prolactina}

Es una hormona producida por la glándula pituitaria anterior, la cual estimula la secreción láctea en la mujer luego del parto, además interviene en los niveles hormonales de los ovarios en las mujeres ${ }^{(3)}$.

La hiperprolactinemia es una de las principales causas de trastornos en la ovulación y de la infertilidad en la mujer; es posible que los niveles elevados de prolactina en la sangre originen un bloqueo en la producción de la hormona luteinizante (LH), imposibilitando la ovulación (4).

Con respecto a los ovarios, la hiperprolactinemia provoca disminución de receptores o de afinidad de $\mathrm{LH}$ en el cuerpo lúteo, lo cual se asocia a una poca producción y secreción de progesterona, puede ser la causa de abortos espontáneos repetidos antes de las 12 semanas de gestación, como consecuencia a una deficiencia lútea ${ }^{(5)}$.

\section{Hormona luteinizante (LH) y resistencia a la insulina (RI)}

En condiciones normales después del ciclo menstrual, la hipófisis en las mujeres comienza a secretar la hormona folículoestimulante (FSH), que ayuda a la producción de estrógenos en el ovario, indispensable para la regeneración del endometrio uterino que se ha eliminado con la menstruación. En la mitad de ciclo ocurre la ovulación por producción del pico de LH y aparece el cuerpo lúteo en el ovario, que segrega progesterona y dispone el endometrio para implantar el posible óvulo fecundado.

La secreción de niveles altos de LH y una secreción de FSH normal o disminuida son una disfunción neuroendocrina. No se han registrado variaciones en neurotransmisores específicos que revelen este trastorno y estudios recientes indican que se trataría eventualmente de una disfunción hipotalámica secundaria a los niveles elevados de andrógenos e insulina.

En las mujeres con SOP se observa un aumento de la producción de la hormona Gonadotropina $(\mathrm{GnRH})$ que produce niveles mayores de $\mathrm{LH}$, lo cual manteniéndose en forma crónica podría promover al hiperandrogenismo y niveles bajos de la hormona folículo estimulante (FSH), lo que no representa lo suficiente para producir la ovulación y la foliculogénesis ${ }^{(5)}$.

En las mujeres con SOP, cuando empieza su ciclo menstrual, los niveles de LH son altos, incluso más que los niveles de FSH, debido a que sus niveles son constantemente altos no se produce la ovulación y los periodos menstruales son irregulares. Durante la noche la producción de LH en el SOP es mayor que en los controles en fase folicular (FF) temprana ${ }^{(6,7)}$.

Elevadas cantidades de insulina origina una mayor secreción de andrógenos por parte del ovario y las glándulas suprarrenales; estimula la secreción de LH como ya se ha descrito y también reduce la producción hepática de la globulina trasportadora de hormonas sexuales (SHBG) con lo cual extiende la cantidad libre y actividad biológica de los andrógenos ${ }^{(6)}$.

La causa por la que se origina una resistencia insulínica en el SOP no está claro. En estas pacientes se ha determinado que no existen cambios en el número o en los receptores de insulina, sino que de las funciones post-receptor en cualquier punto de la señalización insulínica (7).

\section{Resistencia a la insulina}

Un factor importante en la segregación de insulina es el sobrepeso y la obesidad, los niveles elevados de insulina tienen su repercusión en otros órganos como los ovarios, donde la insulina aumenta el cambio de los andrógenos en estrógenos. Los problemas habituales en mujeres con SOP aumentan el riesgo de desarrollar cáncer de endometrio (7).

Todos los ovarios en condiciones normales producen en poca cantidad andrógenos y testosterona. EI SOP se caracteriza cuando los niveles de la hormona LH o los niveles de insulina ayudan a producir un exceso de testosterona ${ }^{(8)}$.

\section{Testosterona}

La esteroidogénesis anormal producida en los ovarios o en las glándulas suprarrenales se considera el primer marcador de SOP. Se ha demostrado un nivel elevado en el plasma de testosterona y dehidroepiandrosterona (DHEA) en pacientes con SOP. Además, existe un aumento de la producción de estradiol por parte de las células ováricas. 
La testosterona y DHT influyen en las alteraciones en el pelo y en su ciclo. Convierten el vello corporal más espeso y oscuro en zonas en donde actúan los andrógenos como cara, cuello, tórax y pubis ${ }^{(8)}$.

La testosterona es el andrógeno más importante en el diagnóstico del hirsutismo y SOP. La testosterona libre es el análisis más sensible para la detección del hiperandrogenismo ${ }^{(9)}$.

\section{Cortisol}

Estudios realizados han demostrado que el ovario posee innervación simpática y receptores de catecolaminas (hormonas que responden al estrés), las cuales al ser estimuladas aumentan la producción de andrógenos y formación de quistes.

Los niveles altos de cortisol se encuentran relacionados con lo psicológico ya que se demostró que las personas con síndrome metabólico tenían mayores niveles de estrés (9).

Se ha estimado que un $50 \%$ de las mujeres con SOP padecen de síndrome metabólico, por lo que es común que sufran de estrés. Se aplicó varios test para medir el nivel de ansiedad, depresión y trastornos emocionales. En estas disfunciones también la producción de cortisol y catecolaminas eran mayores y, por ende, responsables.

En las mujeres adolescentes con SOP la mayor afección emocional se relaciona con el hirsutismo y el acné, mientras que en las mujeres en edad reproductiva la infertilidad es la principal causa de depresión (10).

\section{Factores genéticos del SOP}

SOP es una enfermedad multifactorial, varios genes susceptibles han sido identificados como contribuyentes a la enfermedad como CYP19, CYP17(citocromo P450 17- hidrolasa), CYP11a (citocromo P450 21-hidrolasa), CYP21(citocromo P450 21- hidrolasa) Y FST (folistatina) (11).

\section{CYP19}

Es responsable de la síntesis de la aromatasa, un miembro de la familia de enzimas del citocromo P450 (subfamilia 19) que cataliza la biosíntesis de estrógenos a partir de esteroides $\mathrm{C} 19$. Es inducido por FSH y está presente en varios tejidos diferentes, incluyendo glándulas suprarrenales, músculos, placenta, piel, tejido adiposo y nervioso (12).

Según estudios realizados en mujeres iraníes, se evaluaron 70 casos de mujeres con SOP y 70 como control normal. Registrándose que existen variantes en polimorfismos de CYP19, tomando en cuenta particularmente SNP rs.2414096, evidenciando la asociación del polimorfismo con el gen y el desarrollo de SOP ${ }^{(13)}$.

Otro estudio incluyó 684 individuos (386 pacientes con SOP y 298 controles) y se evaluó la asociación de SNP rs2414096 con SOP, encontrándose que hay una asociación del inicio del SOP a un defecto en la actividad de la aromatasa de CYP19 ${ }^{(13)}$.

\section{CYP17}

El gen CYP17 codifica la 17-alfa-hidroxilasa esteroidea, también conocida como esteroide 17-alfa-monooxigenasa, que interviene tanto en la actividad de la 17-alfa-hidroxilasa como en la de la 17,20-liasa. Estas funciones permiten que las glándulas suprarrenales y las gónadas sinteticen tanto glucocorticoides 17-alfa-hidroxilados. [debe unirse el tanto con el como].

En un estudio se evidenció que la actividad promotora de CYP17 basal y estimulada por forskolina fue 4 veces mayor en células SOP que en células de teca aisladas de ovarios normales. La transcripción del gen CYP17 basal y dependiente de cAMP aumenta en las células de teca SOP; existiendo una regulación diferencial de promotores de genes requeridos para la esteroidogénesis en células de teca con SOP ${ }^{(13)}$.

\section{CYP11}

Codifica la enzima de escisión de la cadena lateral del colesterol (P450scc), iniciando la esteroidogénesis al convertir el colesterol en pregnenolona.

En un estudio de 20 familias con el fenotipo de SOP se examinó la enzima de escisión de la cadena lateral del colesterol CYP11At. Proporcionando evidencia de la vinculación al locus CYP11A. El estudio de asociación se realizó en 97 pacientes caucásicos, identificados consecutivamente con SOP y controles demostrándose una asociación alélica significativa con un polimorfismo repetido de pentanucleótido en la región no traducida 5-prime del gen CYP11A en sujetos con SOP. También se observó que la desregulación de la modificación epigenética por microARN (miARN) que contribuye al desarrollo de la deficiencia de estrógenos, característica de SOP. Dado que existe asociación entre la expresión de miR-320a y el desarrollo de ovocitos en el líquido folicular humano, la comunicación bidireccional existente entre las células del cumulus (CC) y el líquido folicular es esencial para la esteroidogénesis ovárica y los CC son el sitio principal donde finalmente se sintetizan los estrógenos. De esta manera, se demostró. que la desregulación de la cascada miR-320a / RUNX2 / CYP11A1 (CYP19A1) juega un papel importante en el desarrollo de la deficiencia de estrógenos en CC humanos ${ }^{(14)}$.

\section{CYP 21}

Una mayor frecuencia de heterocigosidad para mutaciones en el gen de la 21-hidroxilasa (CYP21) se ha informado en mujeres con SOP y niñas adolescentes con hiperandrogenismo, esta genera una secreción excesiva de andrógenos adrenales en los portadores heterocigóticos obligados de la mutación CYP21 (madres de niños con hiperplasia suprarrenal congénita clásica) tienden a ser 
asintomáticos. Las variantes en los genes implicados en la transducción de señales de insulina podrían representar loci modificadores que aumentan el riesgo de desarrollar SOP. La insulina podría desempeñar un papel en la estimulación directa de la secreción de andrógenos adrenales por la corteza suprarrenal. La unión de la insulina a su receptor inicia una serie de eventos, incluida la fosforilación del receptor de insulina proteínas de sustrato (proteína: IRS; gen: IRS). La proteína IRS-1 juega un papel importante en el esqueleto y en el músculo, mientras que IRS-2 es importante en el hígado, ovario y células pancreáticas. Después de la fosforilación, IRS-1 e IRS-2 se unen y activan efectores posteriores, como la fosfoinositida 3-quinasa (PI 3-quinasa), para promover el metabolismo y acciones mitogénicas de la insulina ${ }^{(15)}$.

\section{Folistatin (FST)}

Follistatin es un regulador intraóvico bien conocido que se une a la activina, una proteína que también se encuentra en el ovario y en parte responsable del crecimiento folicular ${ }^{(15)}$. En un estudio realizado en pacientes con SOP $(n=108)$ y controles sin SOP $(n=20)$. Se observó que las concentraciones más altas de folistatina y concentraciones más bajas de activina A pueden relacionarse con el desarrollo folicular que no avanza más allá de $8-10 \mathrm{~mm}$ y puede ser en parte responsable de la falta de desarrollo folicular preovular en SOP ${ }^{(16)}$.

En otro estudio fueron analizados 37 genes asociados a pacientes con SOP o hiperandrogenemia en 150 familias. La evidencia más fuerte para la vinculación fue con el gen de la folistatina, para el cual las hermanas afectadas mostraron una mayor identidad por descendencia $(72 \%)$, mostrando relación para el desarrollo de SOP ${ }^{(17)}$.

\section{Diagnóstico de SOP}

El SOP al ser un trastorno endocrino de alta prevalencia debe sospecharse en cualquier mujer en edad fértil con hirsutismo, hiperandrogenismo, irregularidades menstruales y obesidad. Es muy importante un diagnóstico oportuno, ya que este síndrome asocia riesgos reproductivos, oncológicos y metabólicos ${ }^{(18)}$.

El cuadro de diagnóstico de SOP tiene una alta variedad en la intensidad de sus manifestaciones generando que su diagnóstico sea dificultoso. La National Institutes of Health (NIH) fue la primera en plantear los criterios de diagnóstico del SOP, estableciendo la hiperandrogenemia y disfunción menstrual como criterios, pero no incluyeron la morfología de ovarios poliquístico en la ecografía (18).

La Sociedad Europea de Reproducción Humana y Embriología y la Sociedad de Medicina Reproductiva evaluaron estos criterios, incorporando la morfología ovárica cómo criterio de diagnóstico en la que incluye la presencia de 12 o más folículos de 2-9 mm de diámetro y un volumen ovárico mayor a $10 \mathrm{~mm}$ en uno o más ovarios ${ }^{(19,20)}$.
En Rotterdan, definen al SOP a aquellas personas que cumplen con dos de tres de estos criterios: hiperandrogenismo clínico o bioquímico (mayor riesgo metabólico), oligoanovulación y la morfología de ovarios poliquístico (21).

Este consenso concluyó que solo que las pacientes con hiperandrogenismo clínico o bioquímico presentan un mayor riesgo metabólico y que, por lo tanto, el diagnóstico de SOP requiere la presencia de hiperandrogenismo (22).

En la adolescencia, la sospecha de SOP representa un motivo de consulta frecuente, esto está dado por las características fisiológicas de este período con los criterios diagnósticos usados para definir este síndrome, tales como ciclos menstruales irregulares, perfil hormonal (tabla 2), hiperandrogenismo y morfología ecográfica de ovario poliquístico ${ }^{(23)}$.

Tabla 2. Valores normales de marcadores hormonales asociados a SOP (24).

\begin{tabular}{cl}
\hline \hline Marcadores hormonales & \multicolumn{1}{c}{ Valor normal } \\
FSH & $3-9 \mathrm{mUl} / \mathrm{ml}$ \\
Prolactina & $2-10 \mathrm{mUl} / \mathrm{ml}$ \\
\hline Testosterona total & 2 a $29 \mathrm{ng} / \mathrm{mL}$ \\
DHEAS & 15 a $70 \mathrm{ng} / \mathrm{dL}$ \\
Estrógenos & 65 a $380 \mu \mathrm{g} / \mathrm{dl}$ \\
SHBG & $27-161 \mathrm{pg} / \mathrm{ml}$ \\
Androstenediona & $40-120 \mathrm{nmol} / \mathrm{L}$ \\
Glucosa & $70-110 \mathrm{mg} / \mathrm{dl}$ \\
Insulina & $60-100 \mathrm{mg} / \mathrm{dl}$ \\
TSH & $0,2-4,7 \mathrm{mUl} / \mathrm{ml}$ \\
Cortisol libre & 6 a $23 \mathrm{mcg} / \mathrm{dL}$ \\
Creatinina & 0.6 a $1.1 \mathrm{mg} / \mathrm{dL}$ \\
\hline \hline
\end{tabular}

En las mujeres adultas se ha establecido la disfunción ovulatoria, el hiperandrogenismo y la morfología del ovario como criterios diagnósticos que en la adolescencia difieren muchos, para algunos autores como Merino ${ }^{(23)}$, debe medirse testosterona total, SHBG, androstenediona, DHEAS, 17-hidroxiprogesterona, prolactina, TSH y perfil lipídico

\section{Diagnóstico ecográfico}

Existen algunas condiciones que debemos tener en cuenta a la hora de evaluar ecográficamente a una paciente con sospecha clínica de SOP ${ }^{25}$. Se debe contar con equipos de ultrasonido adecuados, distintas sondas necesarias para la evaluación, tales como las siguientes:

Sondas convex: para evaluación transabdominal deben oscilar con frecuencias entre $3,5 \mathrm{mHz}$ y $5 \mathrm{mHz}$.

Sondas endocavitarias: para evaluación de la vía transvaginal deben contar con frecuencias entre $6,5 \mathrm{mHz}$ y $8 \mathrm{mHz}$. 
En el diagnostico la exploración se debe realizar en la fase folicular temprana, de preferencia entre los días 3-5 de la menstruación o en cualquier momento si la paciente se encuentra en amenorrea. El estudio no debe ser realizado bajo el uso de anticoncepción hormonal, ya que este modifica la morfología ovárica y si es el caso se debe esperar mínimo 3 meses sin anticoncepción para realizarlo (25).

La vía de preferencia debe ser la transvaginal, ya que es la que permite una mejor resolución, sobre todo en pacientes obesas, sin embargo, se pueden usar otras vías como la transabdominal, transperineal, transintroital o transrectal en pacientes núbiles o en aquellas que por alguna razón anatómica o cultural no se pueda utilizar la vía transvaginal, requiriendo para el uso de estas vías el entrenamiento y la experiencia del médico examinador (26).

Como ya se mencionó antes, los criterios de Rotterdam establecen que la morfología del ovario poliquístico se corresponde con la presencia de al menos 12 folículos cuya medida debe ser entre 2-9 $\mathrm{mm}$ y/o un volumen ovárico incrementado $\geq 10 \mathrm{~mL}$, destacando que la presencia de estos criterios en un solo ovario es suficiente para establecer el diagnóstico. Es importante establecer algunas consideraciones al realizar la evaluación. Si existe un folículo dominante $\mathrm{de} \geq 10 \mathrm{~mm}$ o la presencia de un cuerpo lúteo se debe repetir la exploración en el próximo ciclo entre los 3-5 días. No se tomarán en cuenta la distribución folicular ni la ecogenicidad del estroma ovárico para el diagnóstico. Debemos realizar diagnóstico diferencial con el ovario multifolicular (OMF), en este el tamaño es normal o ligeramente aumentado y contiene 6 o más folículos mayores a $2 \mathrm{~mm}$. Otros factores como la insulina podrían llevar a hiperplasia endometrial y cáncer (27).

\section{Tratamiento para SOP}

\section{Pérdida de peso}

La obesidad es prevalente en mujeres con SOP y se asocia con un empeoramiento de los síntomas. Mujeres con un índice de masa corporal (IMC) igual o mayor de $27 \mathrm{~kg} / \mathrm{m} 2$ son consideradas con obesidad de acuerdo al Consenso Mexicano ${ }^{(28)}$. La pérdida de peso de un 5-7\% con respecto al basal produce un descenso en la concentración circulante de andrógenos, insulina y lípidos, lo cual se acompaña de una mejoría de la sintomatología y de las posibilidades de presentar ciclos ovulatorios ${ }^{(29)}$. Las dietas altas en proteínas pueden tener beneficios adicionales en cuanto a los parámetros endocrinos ${ }^{(30)}$.

\section{Tratamiento hormonal}

Los anticonceptivos hormonales son considerados el tratamiento de primera elección para SOP, ya que combinados proveen varios beneficios: restituyen los ciclos menstruales de manera eficiente, mejoran el hirsutismo en más del 60 $\%$ de los casos por su efecto inhibidor de la LH, lo que aumenta los niveles de SHBG ${ }^{(31)}$. También suprimen el metabolismo de los andrógenos en las adrenales y disminuyen el número de receptores de la 5a-reductasa en la piel, lo que beneficia al acné (32). El tratamiento debe iniciarse con dosis bajas que se incrementarán de acuerdo a las necesidades. Deben evitarse las formulaciones que contienen norgestrel y levonorgestrel por su actividad androgénica ${ }^{(33)}$. En general, las progestinas protegen al endometrio oponiéndose a los efectos proliferativos de los estrógenos, pero tienen un efecto negativo en los parámetros metabólicos ya que aumentan la resistencia a la insulina y los niveles de triglicéridos ${ }^{(34)}$

\section{Agentes sensibilizadores de la insulina}

Está documentado que la disminución en los niveles de insulina mediante el uso de este grupo de fármacos se acompaña de una mejoría del cuadro clínico y de las anormalidades metabólicas. Particularmente, en mujeres obesas con SOP han demostrado ser efectivos y pudieran ser considerados dentro de las opciones de elección para el inicio del manejo ${ }^{(35)}$.

\section{Metformín}

Es un agente anti-hiperglucemiante de la familia de las biguanudas. Actúa principalmente en el hígado inhibiendo parcialmente la gluconeogénesis, lo que reduce en un 17$25 \%$ la producción de glucosa y en el músculo esquelético donde incrementa la captación de glucosa estimulada por insulina en un $29 \%$ y disminuye la oxidación de los ácidos grasos en un 10-20\%. Globalmente esta cadena de eventos provoca en los pacientes diabéticos una mejoría en los niveles de glucosa plasmática en ayuno a la vez que mantiene las concentraciones de insulina sin cambio o incluso con una tendencia a la baja como consecuencia de la mejoría en su accionar ${ }^{(36)}$. En mujeres con SOP el metformín ha demostrado inducir la ovulación y mejorar las posibilidades de lograr un embarazo, incluso en pacientes sometidas a fertilización in vitro por lo que se le considera el tratamiento de segunda línea para SOP ${ }^{(37)}$. La combinación de metformín con anticonceptivos orales o acetato de ciproterona se ha acompañado de una mejoría en los parámetros clínicos y metabólicos (38) (39).

\section{Tratamiento de la anovulación}

El citrato de clomifeno, solo o en combinación con la pérdida de peso, sigue siendo, dada su seguridad y simplicidad, el tratamiento de primera elección en la infertilidad de origen anovulatorio asociado al SOP. Actúa predominantemente como un antiestrógeno uniéndose a los receptores hipotalámicos, lo cual disminuye el mecanismo de retroalimentación negativa ejercida por los estrógenos endógenos. Esto aumenta la secreción de $\mathrm{GnRH}$, la que a su vez estimula la secreción de LH y FSH ${ }^{(40)}$.

La administración de glucocorticoides a bajas dosis puede ser beneficiosa en las mujeres con anovulación hiperandrogénica ${ }^{(41)}$. El mecanismo de acción implica la supresión de la síntesis de andrógenos suprarrenales, lo cual provoca una reducción de hasta el $40 \%$ del total de andrógenos circulantes (42). Igualmente, las dosis bajas de glucocorticoides pueden aumentar la síntesis y secreción de FSH (43). Generalmente se inicia el tratamiento con dexametasona a dosis de 0.25 a $0.5 \mathrm{mg}$ o prednisona 5 $\mathrm{mg}$ durante la noche junto con una progestina para inducir la menstruación antes de restituir el citrato de clomifeno (44). 


\section{Tratamiento quirúrgico}

La resección en cuña bilateral de los ovarios es un procedimiento quirúrgico que se realizó con éxito en pacientes con SOP durante muchos años. Provoca una reducción en los niveles de LH y producción de andrógenos, sin embargo, por las múltiples complicaciones y adherencias relacionadas con el procedimiento ha caído en desuso. Actualmente en casos seleccionados se recurre a la cauterización de los folículos con electrocoagulación ${ }^{(45)}$.

\section{Conclusiones}

El síndrome de ovario poliquístico es la disfunción endocrino-metabólica más frecuente en la mujer; se han identificado genes implicados como el CYP19, CYP11a, CYP17, CYP21 y FST que pueden servir como marcadores. Para el diagnóstico debe existir la presencia de los siguientes condicionantes: oligoanovulación crónica, polisquistosis ovárica por ecografía, hiperandrogenismo clínico o bioquímico. En cuanto al tratamiento de primera línea de tratamiento es el hormonal, la metformin es recomendada en pacientes intolerantes a la glucosa (con diabetes tipo 2), y en pacientes con oligoanovulación que desean embarazarse, el citrato de clomifeno es el tratamiento mejor recomendado.

Conflictos de interés: Los autores declaran que no existen conflicto de interés

\section{Fuentes de financiamiento: Autofinanciado}

\section{REFERENCIAS BIBLIOGRÁFICAS}

1. Jácome F, González C. Síndrome de ovario poliquístico. Rev Mex Med la Reprod Vol [Internet]. 2013;4(2):57-68. Available from: http://www.medigraphic.com/pdfs/reproduccion/mr-2011/mr112b.pdf

2. Santos A, Luque A, Salum R, González J. Síndrome del ovario poliquístico. Volumen. 9, Ciencia Ginecologika. 2005. 54-58 p.

3. Rizzoti K. Genetic regulation of murine pituitary development. J Mol Endocrinol. 2015;54(2):R55-73.

4. Kollmann M, Martins WP, Lima MLS, Craciunas L, Nastri CO, Richardson $A$, et al. Strategies to improve the outcomes of assisted reproduction in women with polycystic ovarian syndrome: a systematic review and meta-analysis. Ultrasound Obstet Gynecol Off J Int Soc Ultrasound Obstet Gynecol [Internet]. 2016; Available from: http://ovidsp.ovid.com/ovidweb. cgi? $=$ JS\&PAGE=reference \&D=medp\&NEWS $=\mathrm{N} \& A N=26924636$

5. Elsersy MAM. Efficacy of Combined Cabergoline and Metformin Compared to Metformin Alone on Cycle Regularity in Patients with Polycystic Ovarian Disease with Hyperprolactinemia: A Randomized Clinical Trial. J Obstet Gynecol India. 2017;67(5):363-9.

6. Vital-Reyes VS, Lopez-Alarcón MG, Inda-Icaza P, Márquez-Maldonado C. Alteraciones metabólicas sutiles en adolescentes con obesidad y síndrome de ovario poliquístico. Gac Med Mex. 2017;153(2):S34-41.

7. Sir-Petermann T, De Guevara AL, Villarroel AC, Preisler J, Echiburú B, Recabarren S. Síndrome de ovario poliquístico y embarazo. Rev Med Chil. 2012;140(7):919-25.

8. Ortiz-Flores AE, Luque-Ramírez M, Escobar-Morreale HF. Polycystic ovary syndrome in adult women. Med Clin (Barcelona) [Internet]. 2019;152(11):450-7. Available from: https://doi.org/10.1016/j.medcli.2018.11.019

9. Padhi T, Garima. Metabolic syndrome and skin: Psoriasis and beyond. Indian J Dermatol [serial online] 2013 [cited 2020 Sep 25];58:299-305. Available from: http://www.e-ijd.org/text.asp?2013/58/4/299/113950

10. Síndrome de Ovario Poliquístico se agravaría por el estrés [Internet]. [cited 2018 Jun 22]. Available from: http://noticias.med.uchile.cl/2007/noviembre/1553-sindrome-de-ovario-poliquistico-se-agravaria-por-el-estres.html
11. Kaur R, Kaur T, Kaur A. Genetic association study from North India to analyze association of CYP19A1 and CYP17A1 with polycystic ovary syndrome. J Assist Reprod Genet [Internet]. 2018 Mar 22 [cited 2018 Jun 22]; Available from: http://www.ncbi.nlm.nih.gov/pubmed/29564739

12. Rui W, Sheng $Y$, Hu R, Miao Y, Han Y, Guo X, et al. Association of Single Nucleotide Polymorphisms in the CYP19A1 Gene with Female Pattern Hair Loss in a Chinese Population. Dermatology [Internet]. 2015 Jul 24 [cited 2018 Jun 22];231(3):239-44. Available from: http://www.ncbi.nlm. nih.gov/pubmed/26228318

13. Afifi L, Saeed L, Pasch LA, Huddleston HG, Cedars MI, Zane LT, et al. Association of ethnicity, Fitzpatrick skin type, and hirsutism: A retrospective cross-sectional study of women with polycystic ovarian syndrome. Int J women's dermatology [Internet]. 2017 Mar [cited 2018 Jun 22];3(1):3743. Available from: http://www.ncbi.nlm.nih.gov/pubmed/28492053

14. Reddy KR, Deepika MLN, Supriya K, Latha KP, Rao SSL, Rani VU, et al. CYP11A1 microsatellite (tttta)n polymorphism in PCOS women from South India. J Assist Reprod Genet. 2014;31(7):857-63.

15. Witchel SF, Kahsar-Miller M, Aston CE, White C, Azziz R. Prevalence of CYP21 mutations and IRS1 variant among women with polycystic ovary syndrome and adrenal androgen excess. Fertil Steril. 2005;83(2):371-5.

16. Teede H, Ng S, Hedger M, Moran L. Follistatin and activins in polycystic ovary syndrome: Relationship to metabolic and hormonal markers. Metabolism [Internet]. 2013 Oct [cited 2018 Jun 22];62(10):1394-400. Available from: http://www.ncbi.nlm.nih.gov/pubmed/23768911

17. Wu C, Wei K, Jiang Z. 5a-reductase activity in women with polycystic ovary syndrome: a systematic review and meta-analysis. Reprod Biol Endocrinol [Internet]. 2017 Mar 27 [cited 2018 Jun 22];15(1):21. Available from: http://www.ncbi.nlm.nih.gov/pubmed/28347315

18. Trivax B, Azziz R. An Update on Polycystic Ovary Syndrome [Internet] Vol. 00, Gynecological Endocrinology. 2007 [cited 2018 Jun 21]. Available from: http://www.touchendocrinology.com/articles/update-polycystic-ovary-syndrome

19. Rotterdam ESHRE/ASRM-Sponsored PCOS Consensus Workshop Group. Revised 2003 consensus on diagnostic criteria and long-term health risks related to polycystic ovary syndrome. Fertil Steril [Internet] 2004 Jan [cited 2018 Jun 21];81(1):19-25. Available from: http://www. ncbi.nlm.nih.gov/pubmed/14711538

20. Merino P, Schulin-Zeuthen C, Codner E. Diagnóstico del Síndrome de Ovario Poliquístico: nuevos fenotipos, nuevas incógnitas. Rev Med Chil [Internet]. 2009 Aug [cited 2018 Jun 21];137(8):1071-80. Available from: http://www.scielo.cl/scielo.php?script=sci_arttext\&pi$\mathrm{d}=$ S0034-98872009000800012\&lng=en\&nrm=iso\&tlng=en

21. Escobar-Morreale HF, Asunción M, Calvo RM, Sancho J, San Millán JL. Receiver operating characteristic analysis of the performance of basal serum hormone profiles for the diagnosis of polycystic ovary syndrome in epidemiological studies. Eur J Endocrinol [Internet]. 2001 Nov [cited 2018 Jun 21];145(5):619-24. Available from: http://www.ncbi.nlm.nih. gov/pubmed/11720881

22. Cho LW, Kilpatrick ES, Jayagopal V, Diver MJ, Atkin SL. Biological variation of total testosterone, free androgen index and bioavailable testosterone in polycystic ovarian syndrome: implications for identifying hyperandrogenaemia. Clin Endocrinol (Oxf) [Internet]. 2007 Sep 20 [cited 2018 Jun 21];0(0):071031175658001-??? Available from: http://www.ncbi. nlm.nih.gov/pubmed/17888022

23. Merino PM, Schulin-Zeuthen PC, Cannoni BG, Conejero RC. Síndrome de ovario poliquístico: diagnóstico en la adolescencia. Rev Médica Clínica Las Condes [Internet]. 2015 Jan 1 [cited 2018 Jun 21];26(1):8893. Available from: https://www.sciencedirect.com/science/article/pii/ S0716864015000140

24. Gutiérrez Cruz, Lozano-Hernández Ricardo, Lozano Carmen-A, Villavicencio Antonio. Tensión arterial y masa corporal en mujeres infértiles con síndrome de ovario poliquístico y su relación con el perfil hormonal. Rev Obstet Ginecol Venez [Internet]. 2014 Sep [citado 2021 Feb 04] ; 74( 3 ): 170-176. Disponible en: http://ve.scielo.org/scielo.php?script=sci_arttext\&pid=S0048-77322014000300005\&lng=es.

25. Diaz A, Laufer MR, Breech LL. Menstruation in girls and adolescents: Using the menstrual cycle as a vital sign. Pediatrics. 2006;118(5):2245- 
50.

26. Sir-Petermann T, Maliqueo Y M, Pérez-Bravo F, Angel B B, Carvajal P F, del Solar P MP, et al. Síndrome de ovario poliquístico: la importancia de establecer su diagnóstico. Rev Med Chil [Internet]. 2001 Jul [cited 2018 Jun 21];129(7):805-12. Available from: http://www.scielo.cl/scielo.php?scrip$\mathrm{t}=$ sci_arttext\&pid=S0034-98872001000700015\&lng=en\&nrm=iso\&tlng=en

27. Ortiz A, Hernández I, Ayala A. Sensibilizantes de la insulina en el tratamiento del ovario poliquístico Artículo de revisión. 2005 [cited 2018 Jun 22];73(6). Available from: http://www.medigraphic.com/pdfs/ginobsmex/ gom-2005/gom056f.pdf

28. Vargas-Carrillo MA, Sánchez-Buenfil G, Herrera-Polanco J, Vargas-Ancona L. Síndrome de ovarios poliquísticos: abordaje diagnóstico y terapéutico. Rev Biomed [Internet]. [cited 2018 Jun 22];14(14):191-203. Available from: http://www.revbiomed.uady.mx/pdf/rb031437.pdf

29. Balen A, Rajkowha M. Polycystic ovary syndrome--a systemic disorder? Best Pract Res Clin Obstet Gynaecol [Internet]. 2003 Apr [cited 2018 Jun 22];17(2):263-74. Available from: http://www.ncbi.nlm.nih.gov/pubmed/12758099

30. Moran LJ, Noakes M, Clifton PM, Tomlinson L, Norman RJ, Norman RJ. Dietary Composition in Restoring Reproductive and Metabolic Physiology in Overweight Women with Polycystic Ovary Syndrome. J Clin Endocrinol Metab [Internet]. 2003 Feb [cited 2018 Jun 22];88(2):812-9. Available from: http://www.ncbi.nlm.nih.gov/pubmed/12574218

31. Poretsky L, Cataldo NA, Rosenwaks Z, Giudice LC. The Insulin-Related Ovarian Regulatory System in Health and Disease. Endocr Rev [Internet]. 1999 Aug [cited 2018 Jun 22];20(4):535-82. Available from: http://www. ncbi.nlm.nih.gov/pubmed/10453357

32. Bledsoe MB, Cobin RH, Futterweit W, Goldzieher JW, Petak SM, Smith KD, et al. American Association of Clinical Endocrinologists medical guidelines for clinical practice for the diagnosis and treatment of Hyperandrogenic Disorders. 120 Endocr Pract [Internet]. 2001 [cited 2018 Jun 22];7(2). Available from: https://www.aace.com/files/hyper-androgenism-2001.pdf

33. Vergara-Quintero F, Díaz-Yúnez I. Revista colombiana de obstetricia y ginecología. [Internet]. Vol. 64, Revista Colombiana de Obstetricia y Ginecología. Federación Colombiana de Obstetricia y Ginecología; 2013 [cited 2018 Jun 22]. 190-198 p. Available from: http://www.scielo.org.co/scielo. php?script=sci_arttext\&pid=S0034-74342013000200009

34. Azziz R, Adashi EY. Stein and Leventhal: 80 years on. Am J Obstet Gynecol [Internet]. 2016 Feb [cited 2018 Jun 22];214(2):247.e1-247.e11. Available from: http://linkinghub.elsevier.com/retrieve/pii/S0002937815025132

35. Nepelska M, Munn S, Landesmann B. AOP Title PPARa activation in utero leading to impaired fertility in males Short name: PPARa activation leading to impaired fertility Summary of the AOP. [cited 2018 Jun 22]; Available from: https://aopwiki.org/wiki/images/f/f9/Aop18-Snapshot-REV-September2015.pdf

36. Caro C, Fuhrer J, Sáez R, Rubio V, Moreno L, Cumsille M. Efectos de la metformina en el síndrome de ovario poliquístico asociado a insulino resistencia. Rev Chil Obstet Ginecol [Internet]. 2002 [cited 2018 Jun 22];67(1):34-40. Available from: http://www.scielo.cl/scielo.php?script=sci_arttext\&pid=S0717-75262002000100007\&lng=en\&nrm=iso\&tlng=en

37. Ibáñez $L$, Valls $C$, Ferrer $A$, Marcos MV, Rodriguez-Hierro $F$, de Zegher $F$. Sensitization to Insulin Induces Ovulation in Nonobese Adolescents with Anovulatory Hyperandrogenism. J Clin Endocrinol Metab [Internet]. 2001 Aug [cited 2018 Jun 22];86(8):3595-8. Available from: http://www.ncbi.nlm. nih.gov/pubmed/11502783

38. Elter K, Imir G, Durmusoglu F. Clinical, endocrine and metabolic effects of metformin added to ethinyl estradiol-cyproterone acetate in non-obese women with polycystic ovarian syndrome: a randomized controlled study. Hum Reprod [Internet]. 2002 Jul [cited 2018 Jun 22];17(7):1729-37. Available from: http://www.ncbi.nlm.nih.gov/pubmed/12093831

39. Ibáñez L, Valls $C$, Ferrer A, Ong K, Dunger DB, de Zegher F. Additive Effects of Insulin-Sensitizing and Anti-Androgen Treatment in Young, Nonobese Women with Hyperinsulinism, Hyperandrogenism, Dyslipidemia, and Anovulation. J Clin Endocrinol Metab [Internet]. 2002 Jun [cited 2018 Jun 22];87(6):2870-4. Available from: http://www.ncbi.nlm.nih.gov/pub$\mathrm{med} / 12050266$
40. Azargoon A, Ghorbani R, Faraji Z. Effects of simvastatin pretreatment on clomiphene response in clomiphene - resistant women with polycystic ovary syndrome. J Fam Reprod Heal [Internet]. 2013 Dec [cited 2018 Jun 22];7(4):165-70. Available from: http://www.ncbi.nlm.nih.gov/pub$\operatorname{med} / 24971120$

41. Singh KB, Dunnihoo DR, Mahajan DK, Bairnsfather LE. Clomiphene-dexamethasone treatment of clomiphene-resistant women with and without the polycystic ovary syndrome. J Reprod Med [Internet]. 1992 Mar [cited 2018 Jun 22];37(3):215-8. Available from: http://www.ncbi. nIm.nih.gov/pubmed/1564704

42. Isaacs JD, Lincoln SR, Cowan BD. Extended clomiphene citrate (CC) and prednisone for the treatment of chronic anovulation resistant to $\mathrm{CC}$ alone. Fertil Steril [Internet]. 1997 Apr [cited 2018 Jun 22];67(4):641-3. Available from: http://www.ncbi.nlm.nih.gov/pubmed/9093187

43. Joffe H, Taylor AE, Hall JE. EDITORIAL: Polycystic Ovarian SyndromeRelationship to Epilepsy and Antiepileptic Drug Therapy. J Clin Endocrinol Metab [Internet]. 2001 Jul 1 [cited 2018 Jun 22];86(7):2946-9. Available from: https://academic.oup.com/jcem/article-lookup/doi/10.1210/ jcem.86.7.7788

44. Katz M, Carr PJ, Cohen BM, Millar RP. Hormonal effects of wedge resection of polycystic ovaries. Obstet Gynecol [Internet]. 1978 Apr [cited 2018 Jun 23];51(4):437-44. Available from: http://www.ncbi.nlm.nih.gov/ pubmed/662227

45. Silva T, Perez J, Amiram MN. Síndrome de ovario poliquístico. diagnóstico y manejo. Rev Médica Clínica Las Condes [Internet]. 2013 [cited 2018 Jun 23];24:818-26. Available from: https://ac.els-cdn.com/ S0716864013702293/1-s2.0-S0716864013702293-main. pdf? tid=1 deff39b-88f1-43fc-b3d7-8cbcd5406c4a\&acdnat $=1529791853$ _0eOf5c879a36337a4702abb6a00cfb08 